\title{
RELIABILITY FOR LAPLACE DISTRIBUTIONS
}

\author{
SARALEES NADARAJAH
}

Received 30 October 2003

In the area of stress-strength models there has been a large amount of work as regards estimation of the reliability $R=\operatorname{Pr}\left(X_{2}<X_{1}\right)$ when $X_{1}$ and $X_{2}$ are independent random variables belonging to the same univariate family of distributions. The algebraic form for $R=\operatorname{Pr}\left(X_{2}<X_{1}\right)$ has been worked out for the majority of the well-known distributions in the standard forms. However, there are still many other distributions (including generalizations of the well-known distributions) for which the form of $R$ has not been derived. In this paper, we consider several Laplace distributions and derive the corresponding forms for the reliability $R$. The calculations involve the use of special functions.

\section{Introduction}

Laplace distributions arise as tractable "lifetime" models in many areas, including life testing and telecommunications. In the context of reliability, the stress-strength model describes the life of a component which has a random strength $X_{1}$ and is subjected to random stress $X_{2}$. The component fails at the instant that the stress applied to it exceeds the strength, and the component will function satisfactorily whenever $X_{1}>X_{2}$. Thus, $R=\operatorname{Pr}\left(X_{2}<X_{1}\right)$ is a measure of component reliability. It has many applications especially in engineering concepts such as structures, deterioration of rocket motors, static fatigue of ceramic components, fatigue failure of aircraft structures, and the aging of concrete pressure vessels. Some examples are the following.

(1) If $X_{2}$ represents the maximum chamber pressure generated by ignition of a solid propellant and $X_{1}$ represents the strength of the rocket chamber, then $R$ is the probability of successful firing of the engine.

(2) If $X_{2}$ represents the diameter of a shaft and $X_{1}$ represents the diameter of a bearing that is to be mounted on the shaft, then $R$ is the probability that the bearing fits without interference.

(3) Let $X_{1}$ and $X_{2}$ be the remission times of two chemicals when they are administered to two kinds of mechanical systems. Inferences about $R$ present a comparison of the effectiveness of the two chemicals. 
(4) The receptor of a communication system operates only if it is stimulated by a source where magnitude $X_{1}$ is greater than a random lower threshold $X_{2}$ for the system. In this case, $R$ is the probability that the receptor operates.

(5) If $X_{1}$ and $X_{2}$ are future observations on the stability of an engineering design, then $R$ would be the predictive probability that $X_{2}$ is less than $X_{1}$. Similarly, if $X_{1}$ and $X_{2}$ represent lifetimes of two electronic devices, then $R$ is the probability that one fails before the other.

(6) If $X_{1}$ represents the distance of a pyrotechnic igniter from its adjacent pellet and $X_{2}$ represents its ignition distance, then $R$ is the probability that the igniter succeeds to bridge the gap in the pyrotechnic chain.

Because of these applications, the calculation and the estimation of $R=\operatorname{Pr}\left(X_{2}<X_{1}\right)$ are important for the class of Laplace distributions. The calculation of $R$ has been extensively investigated in the literature when $X_{1}$ and $X_{2}$ are independent random variables belonging to the same univariate family of distributions. The algebraic form for $R$ has been worked out for the majority of the well-known distributions in their standard forms. These include normal, uniform, exponential, gamma, Weibull, and the Pareto distributions. However, we have identified many other distributions including extensions of the above distributions for which the form of $R$ is not known. Nadarajah [18, 19, 20, 21] and Nadarajah and Kotz [22] have provided comprehensive collections of the forms for $R$ for generalizations of the exponential, gamma, beta, extreme value, logistic, and the Pareto distributions. In this paper, we attempt to do the same for the class of Laplace distributions.

We will assume throughout this paper that $X_{1}$ and $X_{2}$ are continuous and independent random variables. Let $f_{i}$ and $F_{i}$ denote, respectively, the probability density function (pdf) and the cumulative distribution function (cdf) of $X_{i}$. With this notation, one can write

$$
R=\operatorname{Pr}\left(X_{2}<X_{1}\right)=\int_{-\infty}^{\infty} F_{2}(z) f_{1}(z) d z
$$

We will not provide details of the calculations of (1.1) in this paper (they can be obtained from the author). Our calculations make use of a number of special functions. They are the gamma function defined by

$$
\Gamma(a)=\int_{0}^{\infty} z^{a-1} \exp (-z) d z
$$

the incomplete gamma function defined by

$$
\gamma(a, x)=\int_{0}^{x} t^{a-1} \exp (-t) d t
$$

the complementary incomplete gamma function defined by

$$
\Gamma(a, x)=\int_{x}^{\infty} t^{a-1} \exp (-t) d t
$$


and the Gauss hypergeometric function defined by

$$
{ }_{2} F_{1}(a, b ; c ; x)=\sum_{k=0}^{\infty} \frac{(a)_{k}(b)_{k}}{(c)_{k}} \frac{x^{k}}{k !},
$$

where $(a)_{k}=a(a+1) \cdots(a+k-1)$. The properties of these special functions being used can be found in $[9,24,25,26]$.

\section{Standard Laplace distribution}

The standard Laplace distribution has the pdf and the cdf specified by

$$
\begin{aligned}
& f_{i}(x)=\frac{1}{2 \phi_{i}} \exp \left(-\frac{\left|x-\theta_{i}\right|}{\phi_{i}}\right), \\
& F_{i}(x)= \begin{cases}\frac{1}{2} \exp \left(\frac{x-\theta_{i}}{\phi_{i}}\right), & \text { if } x \leq \theta_{i}, \\
1-\frac{1}{2} \exp \left(\frac{\theta_{i}-x}{\phi_{i}}\right), & \text { if } x>\theta_{i},\end{cases}
\end{aligned}
$$

respectively, where $-\infty<x<\infty,-\infty<\theta_{i}<\infty$, and $\phi_{i}>0$. This distribution has been quite commonly used as an alternative to the normal distribution in robustness studies, see, for example, $[2,11]$. It has also attracted interesting applications in the areas of astronomy, biological and environmental sciences, engineering sciences, finance, inventory management, and quality control.

Direct integration using (1.1) shows that the reliability $R$ for the standard Laplace distribution (2.1) and (2.2) is given by

$$
R=\frac{\phi_{1}^{2}}{2\left(\phi_{1}^{2}-\phi_{2}^{2}\right)} \exp \left(\frac{\theta_{1}-\theta_{2}}{\phi_{1}}\right)-\frac{\phi_{2}^{2}}{2\left(\phi_{1}^{2}-\phi_{2}^{2}\right)} \exp \left(\frac{\theta_{1}-\theta_{2}}{\phi_{2}}\right)
$$

if $\theta_{1} \leq \theta_{2}$ and by

$$
R=1+\frac{\phi_{1}^{2}}{2\left(\phi_{2}^{2}-\phi_{1}^{2}\right)} \exp \left(\frac{\theta_{2}-\theta_{1}}{\phi_{1}}\right)-\frac{\phi_{2}^{2}}{2\left(\phi_{2}^{2}-\phi_{1}^{2}\right)} \exp \left(\frac{\theta_{2}-\theta_{1}}{\phi_{2}}\right)
$$

if $\theta_{1}>\theta_{2}$.

\section{Skewed Laplace distributions}

In the last several decades, various forms of skewed Laplace distributions have sporadically appeared in the literature. One of the earliest is due to McGill [17], who considered distributions with the pdf given by

$$
f_{i}(x)= \begin{cases}\frac{1}{2 \psi_{i}} \exp \left(\frac{x-\theta_{i}}{\psi_{i}}\right), & \text { if } x \leq \theta_{i} \\ \frac{1}{2 \phi_{i}} \exp \left(\frac{\theta_{i}-x}{\phi_{i}}\right), & \text { if } x>\theta_{i}\end{cases}
$$


where $-\infty<x<\infty,-\infty<\theta_{i}<\infty, \phi_{i}>0$, and $\psi_{i}>0$. The corresponding cdf is

$$
F_{i}(x)= \begin{cases}\frac{1}{2} \exp \left(\frac{x-\theta_{i}}{\psi_{i}}\right), & \text { if } x \leq \theta_{i} \\ 1-\frac{1}{2} \exp \left(\frac{\theta_{i}-x}{\phi_{i}}\right), & \text { if } x>\theta_{i}\end{cases}
$$

These distributions are also known as the two-piece double exponential. The standard Laplace distribution arises as the particular case of (3.1) and (3.2) for $\phi_{i}=\psi_{i}, i=1,2$. The reliability $R$ for (3.1) and (3.2) turns out to be

$$
\begin{aligned}
R= & \frac{1}{4}\left(2+\frac{\psi_{2}}{\phi_{1}-\psi_{2}}-\frac{\phi_{2}}{\phi_{1}+\phi_{2}}\right) \exp \left(\frac{\theta_{1}-\theta_{2}}{\phi_{1}}\right) \\
& +\frac{1}{4}\left(\frac{\psi_{2}}{\psi_{1}+\psi_{2}}-\frac{\psi_{2}}{\phi_{1}-\psi_{2}}\right) \exp \left(\frac{\theta_{1}-\theta_{2}}{\psi_{2}}\right)
\end{aligned}
$$

when $\theta_{1} \leq \theta_{2}$ and

$$
\begin{aligned}
R=1 & -\frac{1}{4}\left(2+\frac{\phi_{2}}{\phi_{2}-\psi_{1}}-\frac{\psi_{2}}{\psi_{1}+\psi_{2}}\right) \exp \left(\frac{\theta_{2}-\theta_{1}}{\psi_{1}}\right) \\
& -\frac{1}{4}\left(\frac{\phi_{2}}{\phi_{1}+\phi_{2}}+\frac{\phi_{2}}{\phi_{2}-\psi_{1}}\right) \exp \left(\frac{\theta_{2}-\theta_{1}}{\phi_{2}}\right)
\end{aligned}
$$

when $\theta_{1}>\theta_{2}$. Note that (3.3) and (3.4) reduce to (2.3) and (2.4), respectively, when $\phi_{i}=\psi_{i}, i=1,2$.

A variation of (3.1) and (3.2) studied by Holla and Bhattacharya [12] has the pdf

$$
f_{i}(x)= \begin{cases}p_{i} \phi_{i} \exp \left\{\phi_{i}\left(\theta_{i}-x\right)\right\}, & \text { if } x \leq \theta_{i} \\ \left(1-p_{i}\right) \phi_{i} \exp \left\{\phi_{i}\left(x-\theta_{i}\right)\right\}, & \text { if } x>\theta_{i}\end{cases}
$$

where $-\infty<x<\infty,-\infty<\theta_{i}<\infty, \phi_{i}>0$, and $0<p_{i}<1$. Holla and Bhattacharya used this distribution as the compounding distribution of the expected value of a normal distribution. The cdf corresponding to (3.5) is

$$
F_{i}(x)= \begin{cases}p_{i} \exp \left(-\theta_{i} \phi_{i}\right)\left\{\exp \left(\phi_{i} x\right)-1\right\}, & \text { if } x \leq \theta_{i} \\ 1-p_{i} \exp \left(-\theta_{i} \phi_{i}\right)-\left(1-p_{i}\right) \exp \left\{\phi_{i}\left(\theta_{i}-x\right)\right\}, & \text { if } x>\theta_{i}\end{cases}
$$

and standard calculations using (1.1) show that the reliability $R$ is given by

$$
\begin{aligned}
R= & p_{1} \exp \left(-\theta_{1} \phi_{1}\right)-p_{2} \exp \left(-\theta_{2} \phi_{2}\right) \\
& +\left(1-p_{1}\right)\left\{1-\frac{p_{2} \phi_{1}}{\phi_{1}-\phi_{2}}-\frac{\left(1-p_{2}\right) \phi_{1}}{\phi_{1}+\phi_{2}}\right\} \exp \left\{\phi_{1}\left(\theta_{1}-\theta_{2}\right)\right\} \\
& +p_{2} \phi_{1}\left(\frac{p_{1}}{\phi_{1}+\phi_{2}}+\frac{1-p_{1}}{\phi_{1}-\phi_{2}}\right) \exp \left\{\phi_{2}\left(\theta_{1}-\theta_{2}\right)\right\} \\
& -p_{1} p_{2} \exp \left\{-\left(\theta_{1} \phi_{1}+\theta_{2} \phi_{2}\right)\right\}
\end{aligned}
$$


if $\theta_{1} \leq \theta_{2}$ and by

$$
\begin{aligned}
R= & 1+p_{1} \exp \left(-\theta_{1} \phi_{1}\right)-p_{2} \exp \left(-\theta_{2} \phi_{2}\right) \\
& +p_{1}\left\{\frac{p_{2} \phi_{1}}{\phi_{1}+\phi_{2}}+\frac{\left(1-p_{2}\right) \phi_{1}}{\phi_{1}-\phi_{2}}-1\right\} \exp \left\{\phi_{1}\left(\theta_{2}-\theta_{1}\right)\right\} \\
& -\left(1-p_{2}\right) \phi_{1}\left(\frac{p_{1}}{\phi_{1}-\phi_{2}}+\frac{1-p_{1}}{\phi_{1}+\phi_{2}}\right) \exp \left\{\phi_{2}\left(\theta_{2}-\theta_{1}\right)\right\} \\
& -p_{1} p_{2} \exp \left\{-\left(\theta_{1} \phi_{1}+\theta_{2} \phi_{2}\right)\right\}
\end{aligned}
$$

if $\theta_{1}>\theta_{2}$.

Poiraud-Casanova and Thomas-Agnan [23] exploited a skewed Laplace distribution with the pdf

$$
f_{i}(x)=\alpha_{i}\left(1-\alpha_{i}\right) \begin{cases}\exp \left\{\left(1-\alpha_{i}\right)\left(x-\theta_{i}\right)\right\}, & \text { if } x \leq \theta_{i} \\ \exp \left\{\alpha_{i}\left(\theta_{i}-x\right)\right\}, & \text { if } x>\theta_{i}\end{cases}
$$

(where $-\infty<x<\infty,-\infty<\theta_{i}<\infty$, and $0<\alpha_{i}<1$ ) to show the equivalence of certain quantile estimators. The cdf corresponding to (3.9) is

$$
F_{i}(x)= \begin{cases}\alpha_{i} \exp \left\{\left(1-\alpha_{i}\right)\left(x-\theta_{i}\right)\right\}, & \text { if } x \leq \theta_{i}, \\ 1-\left(1-\alpha_{i}\right) \exp \left\{\alpha_{i}\left(\theta_{i}-x\right)\right\}, & \text { if } x>\theta_{i},\end{cases}
$$

and in this case, the reliability $R$ takes the following forms:

$$
\begin{aligned}
R= & \left(1-\alpha_{1}\right)\left\{1+\frac{\alpha_{1} \alpha_{2}}{1-\alpha_{1}-\alpha_{2}}-\frac{\alpha_{1}\left(1-\alpha_{2}\right)}{\alpha_{1}+\alpha_{2}}\right\} \exp \left\{\alpha_{1}\left(\theta_{1}-\theta_{2}\right)\right\} \\
& -\frac{\alpha_{1} \alpha_{2}\left(1-\alpha_{1}\right)}{\left(1-\alpha_{1}-\alpha_{2}\right)\left(2-\alpha_{1}-\alpha_{2}\right)} \exp \left\{\left(1-\alpha_{2}\right)\left(\theta_{1}-\theta_{2}\right)\right\}
\end{aligned}
$$

if $\theta_{1} \leq \theta_{2}$ and

$$
\begin{aligned}
R= & 1+\alpha_{1}\left\{\frac{\left(1-\alpha_{1}\right)\left(1-\alpha_{2}\right)}{1-\alpha_{1}-\alpha_{2}}+\frac{\left(1-\alpha_{1}\right) \alpha_{2}}{2-\alpha_{1}-\alpha_{2}}-1\right\} \exp \left\{\left(1-\alpha_{1}\right)\left(\theta_{2}-\theta_{1}\right)\right\} \\
& -\frac{\alpha_{1}\left(1-\alpha_{1}\right)\left(1-\alpha_{2}\right)}{\left(\alpha_{1}+\alpha_{2}\right)\left(1-\alpha_{1}-\alpha_{2}\right)} \exp \left\{\alpha_{2}\left(\theta_{2}-\theta_{1}\right)\right\}
\end{aligned}
$$

if $\theta_{1}>\theta_{2}$.

Another manner of introducing skewness into a symmetric distribution has been proposed by Fernández and Steel [8]. Here the idea is to convert a symmetric pdf into a skewed one by postulating inverse scale factors in the positive and negative orthants. 
From the standard Laplace pdf (2.1), one obtains the three-parameter family with the pdf

$$
f_{i}(x)=\frac{k_{i}}{\sigma_{i}\left(1+k_{i}^{2}\right)} \begin{cases}\exp \left\{\frac{x-\theta_{i}}{\sigma_{i} k_{i}}\right\}, & \text { if } x \leq \theta_{i} \\ \exp \left\{\frac{k_{i}\left(\theta_{i}-x\right)}{\sigma_{i}}\right\}, & \text { if } x>\theta_{i}\end{cases}
$$

where $-\infty<x<\infty,-\infty<\theta_{i}<\infty, \sigma_{i}>0$, and $k_{i}>0$. This distribution was originally introduced by Hinkley and Revankar [10] and has shown promise in financial modeling. The cdf corresponding to (3.13) is

$$
F_{i}(x)=\frac{1}{1+k_{i}^{2}} \begin{cases}k_{i}^{2} \exp \left(\frac{x-\theta_{i}}{\sigma_{i} k_{i}}\right), & \text { if } x \leq \theta_{i}, \\ 1+k_{i}^{2}-\exp \left(\frac{k_{i}\left(\theta_{i}-x\right)}{\sigma_{i}}\right), & \text { if } x>\theta_{i}\end{cases}
$$

and in this case, the reliability $R$ takes the following forms:

$$
\begin{aligned}
R= & \left\{\frac{1}{1+k_{1}^{2}}+\frac{\sigma_{1} \sigma_{2} k_{2}^{3}}{\left(1+k_{2}^{2}\right)\left(\sigma_{1}-k_{1} k_{2} \sigma_{2}\right)}-\frac{\sigma_{1} \sigma_{2}}{\left(1+k_{2}^{2}\right)\left(\sigma_{1} k_{2}+\sigma_{2} k_{1}\right)}\right\} \exp \left\{\frac{k_{1}\left(\theta_{1}-\theta_{2}\right)}{\sigma_{1}}\right\} \\
& +\frac{\sigma_{1} \sigma_{2} k_{2}^{3}}{1+k_{2}^{2}}\left\{\frac{k_{1}}{\sigma_{1} k_{1}+\sigma_{2} k_{2}}-\frac{1}{\sigma_{1}-k_{1} k_{2} \sigma_{2}}\right\} \exp \left\{\frac{\theta_{1}-\theta_{2}}{\sigma_{2} k_{2}}\right\}
\end{aligned}
$$

if $\theta_{1} \leq \theta_{2}$ and

$$
\begin{aligned}
R= & 1+k_{1}\left\{\frac{\sigma_{1} \sigma_{2} k_{2}^{3}}{\left(1+k_{2}^{2}\right)\left(\sigma_{1} k_{1}+\sigma_{2} k_{2}\right)}+\frac{\sigma_{1} \sigma_{2}}{\left(1+k_{2}^{2}\right)\left(\sigma_{2}-\sigma_{1} k_{1} k_{2}\right)}-\frac{k_{1}}{1+k_{1}^{2}}\right\} \exp \left\{\frac{\theta_{2}-\theta_{1}}{\sigma_{1} k_{1}}\right\} \\
& -\frac{\sigma_{1} \sigma_{2}}{1+k_{2}^{2}}\left(\frac{k_{1}}{\sigma_{2}-\sigma_{1} k_{1} k_{2}}+\frac{1}{\sigma_{2} k_{1}+\sigma_{1} k_{2}}\right) \exp \left\{\frac{k_{2}\left(\theta_{2}-\theta_{1}\right)}{\sigma_{2}}\right\}
\end{aligned}
$$

if $\theta_{1}>\theta_{2}$.

The most recent skewed Laplace distribution has been studied by Aryal and Nadarajah [3]. A random variable $X$ is said to have the skewed Laplace distribution if its pdf is $f(x)=2 g(x) G(\lambda x), \lambda>0$, where $g$ and $G$ are, respectively, the pdf and the cdf of the standard Laplace distribution. It follows then that $f(x)$ is given by

$$
f_{i}(x)= \begin{cases}\frac{1}{2 \phi_{i}} \exp \left\{\frac{\left(1+\lambda_{i}\right) x}{\phi_{i}}\right\}, & \text { if } x \leq 0, \\ \frac{1}{\phi_{i}} \exp \left(-\frac{x}{\phi_{i}}\right) \exp \left\{1-\frac{1}{2} \exp \left(-\frac{\lambda_{i} x}{\phi_{i}}\right)\right\}, & \text { if } x>0,\end{cases}
$$


where $-\infty<x<\infty, \lambda_{i}>0$, and $\phi_{i}>0$. The main feature of this distribution is that a new parameter $\lambda_{i}$ is introduced to control skewness and kurtosis. The cdf corresponding to (3.17) is

$$
F_{i}(x)= \begin{cases}\frac{1}{2\left(1+\lambda_{i}\right)} \exp \left\{\frac{\left(1+\lambda_{i}\right) x}{\phi_{i}}\right\}, & \text { if } x \leq 0, \\ 1-\left\{1-\frac{1}{2\left(1+\lambda_{i}\right)} \exp \left(-\frac{\lambda_{i} x}{\phi_{i}}\right)\right\} \exp \left(-\frac{x}{\phi_{i}}\right), & \text { if } x>0\end{cases}
$$

and simple calculations using (1.1) show that the reliability $R$ is given by

$$
R=\frac{\phi_{1}}{\phi_{1}+\phi_{2}}-\frac{1}{2\left(1+\lambda_{1}\right)}+\frac{\phi_{2}}{2\left\{\phi_{1}+\left(1+\lambda_{1}\right) \phi_{2}\right\}}+\frac{\phi_{2}}{2\left(1+\lambda_{2}\right)\left\{\left(1+\lambda_{2}\right) \phi_{1}+\phi_{2}\right\}}
$$

Note that if $\phi_{1}=\phi_{2}$, then the above reduces to $R=1 / 2$.

\section{Generalized Laplace distribution}

Subbotin [28] proposed a generalization of the Laplace distribution with the pdf

$$
f_{i}(x)=\frac{1}{2 p_{i}^{1 / p_{i}} \sigma_{p_{i}} \Gamma\left(1+1 / p_{i}\right)} \exp \left(-\frac{\left|x-\mu_{i}\right|^{p_{i}}}{p_{i} \sigma_{p_{i}}^{p_{i}}}\right),
$$

where $-\infty<x<\infty, \mu_{i}=E\left(X_{i}\right)$ is the location parameter, $\sigma_{p_{i}}=\left\{E\left(\left|X_{i}-\mu_{i}\right|^{p_{i}}\right)\right\}^{1 / p_{i}}$ is the scale parameter, and $p_{i}>0$ is the shape parameter. This generalization is sometimes referred to as the exponential power function distribution. This distribution is widely used in Bayesian inference (see, e.g., [6, 30]). Estimation issues related to (4.1) are discussed in $[1,33]$.

Using the definition of the incomplete gamma functions, one can write the cdf corresponding to (4.1) as

$$
F_{i}(x)=\frac{1}{2 \Gamma\left(1 / p_{i}\right)} \begin{cases}\Gamma\left(\frac{1}{p_{i}}, \frac{\left(\mu_{i}-x\right)^{p_{i}}}{p_{i} \sigma_{p_{i}}^{p_{i}}}\right), & \text { if } x \leq \mu_{i}, \\ \Gamma\left(\frac{1}{p_{i}}\right)+\gamma\left(\frac{1}{p_{i}}, \frac{\left(x-\mu_{i}\right)^{p_{i}}}{p_{i} \sigma_{p_{i}}^{p_{i}}}\right), & \text { if } x>\mu_{i} .\end{cases}
$$

The form of (1.1) for (4.1) and (4.2) is difficult to calculate. However, in the particular case $\mu_{1}=\mu_{2}=\mu$, one can write

$$
R=\frac{1}{4}+\frac{I_{1}+I_{2}}{4 p_{1}^{1 / p_{1}} \sigma_{p_{1}} \Gamma\left(1+1 / p_{1}\right) \Gamma\left(1 / p_{2}\right)}
$$


where $I_{1}$ and $I_{2}$ are the integrals

$$
\begin{aligned}
& I_{1}=\int_{-\infty}^{\mu} \exp \left\{-\frac{(\mu-x)^{p_{1}}}{p_{1} \sigma_{p_{1}}^{p_{1}}}\right\} \Gamma\left(\frac{1}{p_{2}}, \frac{(\mu-x)^{p_{2}}}{p_{2} \sigma_{p_{2}}^{p_{2}}}\right) d x, \\
& I_{2}=\int_{\mu}^{\infty} \exp \left\{-\frac{(x-\mu)^{p_{1}}}{p_{1} \sigma_{p_{1}}^{p_{1}}}\right\} \gamma\left(\frac{1}{p_{2}}, \frac{(x-\mu)^{p_{2}}}{p_{2} \sigma_{p_{2}}^{p_{2}}}\right) d x .
\end{aligned}
$$

On substituting $y=(\mu-x)^{p_{2}} /\left(p_{2} \sigma_{p_{2}}^{p_{2}}\right)$ and $y=(x-\mu)^{p_{2}} /\left(p_{2} \sigma_{p_{2}}^{p_{2}}\right)$, the integrals $I_{1}$ and $I_{2}$ reduce to the simpler forms

$$
\begin{aligned}
& I_{1}=p_{2}^{1 / p_{2}-1} \sigma_{p_{2}} \int_{0}^{\infty} y^{1 / p_{2}-1} \exp \left(-\delta y^{p_{1} / p_{2}}\right) \Gamma\left(\frac{1}{p_{2}}, y\right) d y, \\
& I_{2}=p_{2}^{1 / p_{2}-1} \sigma_{p_{2}} \int_{0}^{\infty} y^{1 / p_{2}-1} \exp \left(-\delta y^{p_{1} / p_{2}}\right) \gamma\left(\frac{1}{p_{2}}, y\right) d y,
\end{aligned}
$$

respectively, where $\delta=p_{2}^{p_{1} / p_{2}} \sigma_{p_{2}}^{p_{1}} /\left(p_{1} \sigma_{p_{1}}^{p_{1}}\right)$. When $p_{1} \neq p_{2}$, these simplified integrals can be expressed as infinite sums of gamma functions. In fact, the use of [25, equation (2.10.1.5)] shows that

$$
\begin{aligned}
& I_{1}=p_{2}^{1 / p_{2}} \sigma_{p_{2}} \begin{cases}\frac{\delta^{-1 / p_{1}}}{p_{1}} \Gamma\left(\frac{1}{p_{1}}\right) \Gamma\left(\frac{1}{p_{2}}\right)-\frac{p_{2} \delta^{-2 / p_{1}}}{p_{1}} \sum_{k=0}^{\infty} \frac{(-1)^{k} \delta^{-p_{2} k / p_{1}}}{k !\left(1+p_{2} k\right)} \Gamma\left(\frac{2+p_{2} k}{p_{1}}\right), & \text { if } p_{1}>p_{2}, \\
\frac{\delta^{-1 / p_{1}}}{p_{1}} \Gamma\left(\frac{1}{p_{1}}\right) \Gamma\left(\frac{1}{p_{2}}\right)+\sum_{k=0}^{\infty} \frac{(-1)^{k} \delta^{k}}{k !\left(1+p_{1} k\right)} \Gamma\left(\frac{1}{p_{1}}+\frac{1+p_{1} k}{p_{2}}\right), & \text { if } p_{1}<p_{2},\end{cases} \\
& I_{2}=p_{2}^{1 / p_{2}} \sigma_{p_{2}} \begin{cases}\frac{p_{2}}{p_{1} \delta^{2 / p_{1}}} \sum_{k=0}^{\infty} \frac{(-1)^{k} \delta^{-p_{2} k / p_{1}}}{k !\left(1+p_{2} k\right)} \Gamma\left(\frac{2+p_{2} k}{p_{1}}\right), & \text { if } p_{1}>p_{2}, \\
-\sum_{k=0}^{\infty} \frac{(-1)^{k} \delta^{k}}{k !\left(1+p_{1} k\right)} \Gamma\left(\frac{1}{p_{1}}+\frac{1+p_{1} k}{p_{2}}\right), & \text { if } p_{1}<p_{2} .\end{cases}
\end{aligned}
$$

In the particular case $p_{1}=p_{2}=p$, the integrals $I_{1}$ and $I_{2}$ can be expressed in terms of the Gauss hypergeometric function. Application of [25, equation (2.10.3.2)] shows that

$$
\begin{aligned}
& I_{1}=p^{1 / p-1} \sigma_{p} \delta^{-2 / p}\left[\delta^{1 / p}\left\{\Gamma\left(\frac{1}{p}\right)\right\}^{2}-p \Gamma\left(\frac{2}{p}\right){ }_{2} F_{1}\left(\frac{1}{p}, \frac{2}{p} ; 1+\frac{1}{p} ;-\frac{1}{\delta}\right)\right], \\
& I_{2}=p^{1 / p} \sigma_{p} \delta^{-2 / p} \Gamma\left(\frac{2}{p}\right){ }_{2} F_{1}\left(\frac{1}{p}, \frac{2}{p} ; 1+\frac{1}{p} ;-\frac{1}{\delta}\right) .
\end{aligned}
$$

The corresponding expressions for the reliability $R$ follow by substituting (4.6) and (4.7) into (4.3). 


\section{Reflected gamma distribution}

Borghi [5] introduced the reflected gamma distribution specified by the pdf

$$
f_{i}(x)=\frac{1}{2 \phi_{i} \Gamma\left(\alpha_{i}\right)}\left|\frac{x-\theta_{i}}{\phi_{i}}\right|^{\alpha_{i}-1} \exp \left\{-\left|\frac{x-\theta_{i}}{\phi_{i}}\right|\right\},
$$

where $-\infty<x<\infty,-\infty<\theta_{i}<\infty, \alpha_{i}>0$, and $\phi_{i}>0$. This includes the standard Laplace distribution as a particular case for $\alpha_{i}=1$. Estimation issues related to (5.1) have been studied by Kantam and Narasimham [15].

Using the definition of the incomplete gamma functions, one can write the cdf corresponding to (5.1) as

$$
F_{i}(x)=\frac{1}{2 \Gamma\left(\alpha_{i}\right)} \begin{cases}\Gamma\left(\alpha_{i}, \frac{\theta_{i}-x}{\phi_{i}}\right), & \text { if } x \leq \theta_{i}, \\ \Gamma\left(\alpha_{i}\right)+\gamma\left(\alpha_{i}, \frac{x-\theta_{i}}{\phi_{i}}\right), & \text { if } x>\theta_{i} .\end{cases}
$$

Following the approach in Section 4, one writes the associated reliability $R$ as

$$
R=\frac{1}{4}+\frac{I_{1}+I_{2}}{4 \phi_{1}^{\alpha_{1}} \Gamma\left(\alpha_{1}\right) \Gamma\left(\alpha_{2}\right)},
$$

where

$$
\begin{aligned}
& I_{1}=\phi_{2}^{\alpha_{1}} \int_{0}^{\infty} y^{\alpha_{1}-1} \exp \left(-\frac{\phi_{2} y}{\phi_{1}}\right) \Gamma\left(\alpha_{2}, y\right) d y \\
& I_{2}=\phi_{2}^{\alpha_{1}} \int_{0}^{\infty} y^{\alpha_{1}-1} \exp \left(-\frac{\phi_{2} y}{\phi_{1}}\right) \gamma\left(\alpha_{2}, y\right) d y
\end{aligned}
$$

Utilizing [25, equation (2.10.3.2)], these integrals can be expressed in terms of the Gauss hypergeometric function. It follows that

$$
\begin{aligned}
& I_{1}=\phi_{1}^{\alpha_{1}} \Gamma\left(\alpha_{1}\right) \Gamma\left(\alpha_{2}\right)-\frac{\phi_{1}^{\alpha_{1}+\alpha_{2}} \Gamma\left(\alpha_{1}+\alpha_{2}\right)}{\alpha_{2} \phi_{2}^{\alpha_{2}}}{ }_{2} F_{1}\left(\alpha_{2}, \alpha_{1}+\alpha_{2} ; 1+\alpha_{2} ;-\frac{\phi_{1}}{\phi_{2}}\right), \\
& I_{2}=\frac{\phi_{1}^{\alpha_{1}+\alpha_{2}} \Gamma\left(\alpha_{1}+\alpha_{2}\right)}{\alpha_{2} \phi_{2}^{\alpha_{2}}}{ }_{2} F_{1}\left(\alpha_{2}, \alpha_{1}+\alpha_{2} ; 1+\alpha_{2} ;-\frac{\phi_{1}}{\phi_{2}}\right) .
\end{aligned}
$$

The expression for the reliability $R$ follows by substituting (5.5) into (5.3).

\section{Double Weibull distribution}

Balakrishnan and Kocherlakota [4] introduced the double Weibull distribution specified by the pdf

$$
f_{i}(x)=\frac{c_{i}}{2 \phi_{i}}\left|\frac{x-\theta_{i}}{\phi_{i}}\right|^{c_{i}-1} \exp \left\{-\left|\frac{x-\theta_{i}}{\phi_{i}}\right|^{c_{i}}\right\},
$$


where $-\infty<x<\infty,-\infty<\theta_{i}<\infty, \phi_{i}>0$, and $c_{i}>0$. This includes the standard Laplace distribution as a particular case for $c_{i}=1$. Estimation issues related to (6.1) have been studied by Balakrishnan and Kocherlakota [4], Dattatreya Rao and Narasimham [7], and Vasudeva Rao et al. [31].

Direct integration shows that the cdf corresponding to (6.1) is given by

$$
F_{i}(x)= \begin{cases}\frac{1}{2} \exp \left\{-\left(\frac{\theta_{i}-x}{\phi_{i}}\right)^{c_{i}}\right\}, & \text { if } x \leq \theta_{i}, \\ 1-\frac{1}{2} \exp \left\{-\left(\frac{x-\theta_{i}}{\phi_{i}}\right)^{c_{i}}\right\}, & \text { if } x>\theta_{i} .\end{cases}
$$

The general form of the reliability $R$ associated with (6.1) and (6.2) is difficult to calculate. However, in the particular case $\theta_{1}=\theta_{2}$, one can easily show that $R=1 / 2$.

\section{Sargan distribution}

Sargan distribution arises by summing $n+1$ independent and identically distributed standard Laplace random variables. Its pdf takes the form

$$
f_{i}(x)=\frac{\alpha_{i}}{2} \exp \left\{-\alpha_{i}\left|x-\theta_{i}\right|\right\} \sum_{k=0}^{n_{i}} \gamma_{k}^{(i)} \alpha_{i}^{k}\left|x-\theta_{i}\right|^{k}
$$

where $-\infty<x<\infty,-\infty<\theta_{i}<\infty, \alpha_{i}>0$, and

$$
\gamma_{k}^{(i)}=\frac{\left(2 n_{i}-k\right) ! 2^{k-2 n_{i}}}{n_{i} ! k !\left(n_{i}-k\right) !} .
$$

This distribution is also a particular case of the Bessel function distribution. The cdf corresponding to (7.1) can be expressed in terms of the complementary incomplete gamma function:

$$
F_{i}(x)=\left\{\begin{array}{cl}
\frac{\exp \left(-\alpha_{i} \theta_{i}\right)}{2} \sum_{j=0}^{n_{i}} \gamma_{j}^{(i)} \sum_{k=0}^{j}\left(\begin{array}{l}
j \\
k
\end{array}\right)\left(\alpha_{i} \theta_{i}\right)^{j-k} \Gamma\left(k+1,-\alpha_{i} x\right), & \text { if } x \leq \theta_{i}, \\
F\left(\theta_{i}\right)+\frac{\exp \left(\alpha_{i} \theta_{i}\right)}{2} \sum_{j=0}^{n_{i}} \gamma_{j}^{(i)} \sum_{k=0}^{j}\left(\begin{array}{l}
j \\
k
\end{array}\right)\left(-\alpha_{i} \theta_{i}\right)^{k} & \\
\times\left\{\Gamma\left(j-k+1, \alpha_{i} \theta_{i}\right)-\Gamma\left(j-k+1, \alpha_{i} x\right)\right\}, & \text { if } x>\theta_{i} .
\end{array}\right.
$$

The reliability $R$ associated with (7.1) and (7.3) cannot be calculated in closed form if $\theta_{i} \neq 0$. However, if $\theta_{1}=\theta_{2}=0$, then one can easily obtain the following neat expression:

$$
R=\left(1-\frac{1}{2} \sum_{j=0}^{n_{1}} \gamma_{j}^{(1)} j !\right) \sum_{j=0}^{n_{2}} \gamma_{j}^{(2)} j !
$$




\section{Compound Laplace gamma distribution}

One of the most popular compound Laplace distributions is the compound Laplace gamma distribution given by the pdf

$$
f_{i}(x)=\frac{\alpha_{i} \beta_{i}}{2}\left\{1+\beta_{i}\left|x-\theta_{i}\right|\right\}^{-\left(\alpha_{i}+1\right)}
$$

where $-\infty<x<\infty,-\infty<\theta_{i}<\infty, \alpha_{i}>0$, and $\beta_{i}>0$. Note that as $\alpha_{i} \rightarrow \infty$ and $\beta_{i} \rightarrow 0$ with $\alpha_{i} \beta_{i}=1$, (8.1) approaches the pdf of the standard Laplace distribution. The cdf corresponding to $(8.1)$ is

$$
F_{i}(x)= \begin{cases}\frac{1}{2}\left\{1+\beta_{i}\left|x-\theta_{i}\right|\right\}^{-\alpha_{i}}, & \text { if } x \leq \theta_{i}, \\ 1-\frac{1}{2}\left\{1+\beta_{i}\left|x-\theta_{i}\right|\right\}^{-\alpha_{i}}, & \text { if } x>\theta_{i}\end{cases}
$$

and using (1.1), one can write the associated reliability $R$ as

$$
R=1-F_{1}\left(\theta_{2}\right)+\frac{\alpha_{1} \beta_{1}}{4}\left(I_{1}+I_{2}-I_{3}\right)
$$

where the integrals $I_{1}, I_{2}$, and $I_{3}$ are given by

$$
\begin{aligned}
& I_{1}=\int_{-\infty}^{\min \left(\theta_{1}, \theta_{2}\right)}\left\{1+\beta_{1}\left(\theta_{1}-x\right)\right\}^{-\left(1+\alpha_{1}\right)}\left\{1+\beta_{2}\left(\theta_{2}-x\right)\right\}^{-\alpha_{2}} d x, \\
& I_{2}=\int_{\min \left(\theta_{1}, \theta_{2}\right)}^{\max \left(\theta_{1}, \theta_{2}\right)}\left\{1+\beta_{1}\left|x-\theta_{1}\right|\right\}^{-\left(1+\alpha_{1}\right)}\left\{1+\beta_{2}\left|x-\theta_{2}\right|\right\}^{-\alpha_{2}} d x, \\
& I_{3}=\int_{\max \left(\theta_{1}, \theta_{2}\right)}^{\infty}\left\{1+\beta_{1}\left(x-\theta_{1}\right)\right\}^{-\left(1+\alpha_{1}\right)}\left\{1+\beta_{2}\left(x-\theta_{2}\right)\right\}^{-\alpha_{2}} d x .
\end{aligned}
$$

The integrals $I_{1}$ and $I_{3}$ can be expressed in terms of the Gauss hypergeometric function by using $\left[9\right.$, equation (3.197.1)]. For instance, if $\theta_{1}<\theta_{2}$, then one can show that

$$
\begin{aligned}
& I_{1}=\frac{\alpha_{1} \beta_{1}\left\{1+\beta_{2}\left(\theta_{2}-\theta_{1}\right)\right\}^{1-\alpha_{2}}}{4\left(\alpha_{1}+\alpha_{2}\right) \beta_{2}} F_{1}\left(1+\alpha_{1}, 1 ; 1+\alpha_{1}+\alpha_{2} ; 1-\frac{\beta_{1}}{\beta_{2}}-\beta_{1}\left(\theta_{2}-\theta_{1}\right)\right), \\
& I_{3}=\frac{\alpha_{1}\left\{1+\beta_{1}\left(\theta_{2}-\theta_{1}\right)\right\}^{-\alpha_{1}}}{4\left(\alpha_{1}+\alpha_{2}\right)}{ }_{2} F_{1}\left(\alpha_{2}, 1 ; 1+\alpha_{1}+\alpha_{2} ; 1-\frac{\beta_{2}}{\beta_{1}}-\beta_{2}\left(\theta_{2}-\theta_{1}\right)\right) .
\end{aligned}
$$

However, the integral $I_{2}$ cannot be simplified further unless of course $\theta_{1}=\theta_{2}$. In this particular case, one gets $R=1 / 2$. 


\section{Laplace normal mixture distribution}

Kanji [14] and Jones and McLachlan [13] introduced the Laplace normal mixture distribution with the pdf

$$
f_{i}(x)=\frac{p_{i}}{2 \phi_{i}} \exp \left(-\frac{\left|x-\theta_{i}\right|}{\phi_{i}}\right)+\frac{1-p_{i}}{\sqrt{2 \pi} \sigma_{i}} \exp \left\{-\frac{\left(x-\theta_{i}\right)^{2}}{2 \sigma_{i}^{2}}\right\},
$$

where $-\infty<x<\infty,-\infty<\theta_{i}<\infty, \phi_{i}>0, \sigma_{i}>0$, and $0<p_{i}<1$. This distribution has been successfully applied to fit wind shear data. Maximum likelihood estimation of the parameters of (9.1) has been discussed by Kapoor and Kanji [16] and Scallan [27].

The cdf and the reliability $R$ corresponding to (9.1) can also be expressed in the mixture forms

$$
\begin{aligned}
F_{i}(x) & = \begin{cases}\frac{p_{i}}{2} \exp \left(\frac{x-\theta_{i}}{\phi_{i}}\right)+\left(1-p_{i}\right) \Phi\left(\frac{x-\theta_{i}}{\sigma_{i}}\right), & \text { if } x \leq \theta_{i} \\
p_{i}-\frac{p_{i}}{2} \exp \left(\frac{\theta_{i}-x}{\phi_{i}}\right)+\left(1-p_{i}\right) \Phi\left(\frac{x-\theta_{i}}{\sigma_{i}}\right), & \text { if } x>\theta_{i}\end{cases} \\
R & =p_{1} p_{2} R_{11}+p_{1}\left(1-p_{2}\right) R_{12}+\left(1-p_{1}\right) p_{2} R_{21}+\left(1-p_{1}\right)\left(1-p_{2}\right) R_{22}
\end{aligned}
$$

where $\Phi(\cdot)$ denotes the cdf of the standard normal distribution, $R_{11}$ and $R_{22}$ denote the reliability $R$ for the standard Laplace and normal distributions, respectively, and $R_{12}$ and $R_{21}$ are given by

$$
\begin{aligned}
R_{12}= & \frac{1}{2 \phi_{1}} \int_{-\infty}^{\infty} \exp \left(-\frac{\left|x-\theta_{1}\right|}{\phi_{1}}\right) \Phi\left(\frac{x-\theta_{2}}{\sigma_{2}}\right) d x \\
R_{21}= & \frac{1}{2 \sqrt{2 \pi} \sigma_{1}} \int_{-\infty}^{\theta_{2}} \exp \left\{\frac{x-\theta_{2}}{\phi_{2}}-\frac{\left(x-\theta_{1}\right)^{2}}{2 \sigma_{1}^{2}}\right\} d x \\
& +\frac{1}{\sqrt{2 \pi} \sigma_{1}} \int_{\theta_{2}}^{\infty}\left\{1-\frac{1}{2} \exp \left(\frac{\theta_{2}-x}{\phi_{2}}\right)\right\} \exp \left\{-\frac{\left(x-\theta_{1}\right)^{2}}{2 \sigma_{1}^{2}}\right\} d x
\end{aligned}
$$

respectively. The expression for $R_{11}$ is already calculated in (2.3) and (2.4). Expressions for $R_{22}$ are widely available in the literature (see, e.g., [32]). It is known, for instance, that if $\sigma_{1}=\sigma_{2}=\sigma$, then

$$
R_{22}=\frac{1}{2}+\frac{1}{2}\left\{\Phi^{2}\left(\frac{\theta_{2}-\theta_{1}}{\sqrt{2} \sigma}\right)+\Phi^{2}\left(\frac{\theta_{1}-\theta_{2}}{\sqrt{2} \sigma}\right)\right\} .
$$

Also, if $\theta_{1}=\theta_{2}$, then it is known that $R_{22}=1 / 2$. An expression for $R_{12}$ can be evaluated by an easy application of [25, equation (2.8.9.1)]. It turns out that

$$
\begin{aligned}
R_{12}= & \frac{1}{2}+\frac{1}{2} \exp \left\{\frac{\sigma_{2}^{2}-2 \phi_{1}\left(\theta_{1}-\theta_{2}\right)}{2\left(\theta_{1}-\theta_{2}\right)^{2}}\right\} \Phi\left(\frac{\sigma_{2}}{\theta_{2}-\theta_{1}}+\frac{\phi_{1}}{\sigma_{2}}\right) \\
& +\frac{1}{2} \exp \left\{\frac{\sigma_{2}^{2}+2 \phi_{1}\left(\theta_{1}-\theta_{2}\right)}{2\left(\theta_{1}-\theta_{2}\right)^{2}}\right\} \Phi\left(\frac{\sigma_{2}}{\theta_{2}-\theta_{1}}-\frac{\phi_{1}}{\sigma_{2}}\right) .
\end{aligned}
$$


Direct integration yields an expression for $R_{21}$ given by

$$
\begin{aligned}
R_{21}= & 1-\Phi\left(\frac{\theta_{2}-\theta_{1}}{\sigma_{1}}\right)+\frac{1}{2} \exp \left\{\frac{\sigma_{1}^{2}+2 \phi_{2}\left(\theta_{1}-\theta_{2}\right)}{2 \phi_{2}^{2}}\right\} \Phi\left(\frac{\theta_{2}-\theta_{1}}{\sigma_{1}}-\frac{\sigma_{1}}{\phi_{2}}\right) \\
& -\frac{1}{2} \exp \left\{\frac{\sigma_{1}^{2}+2 \phi_{2}\left(\theta_{2}-\theta_{1}\right)}{2 \phi_{2}^{2}}\right\}\left\{1-\Phi\left(\frac{\theta_{2}-\theta_{1}}{\sigma_{1}}+\frac{\sigma_{1}}{\phi_{2}}\right)\right\} .
\end{aligned}
$$

Substituting (9.6), (9.7), and the known expressions for $R_{11}$ and $R_{22}$ into (9.3), one obtains an expression for $R$.

\section{Translated Laplace distributions}

Tadikamalla and Johnson [29] proposed three translated families of Laplace distributions. If $Y$ has the standard Laplace distribution, then the three distributions can be specified by the following translations:

(1) $Y=\log \beta+\alpha \log X$ for $\alpha>0$ and $\beta>0$, then $X$ is said to have the log-Laplace distribution,

(2) $Y=\log \beta+\alpha \log \left(X^{*} /\left(1-X^{*}\right)\right)$ for $\alpha>0$ and $\beta>0$, then $X^{*}$ is said to have the $L_{B}$ system distribution,

(3) $Y=\log \beta+\alpha \operatorname{arcsinh} X^{* *}$ for $\alpha>0$ and $\beta>0$, then $X^{* *}$ is said to have the $L_{U}$ system distribution.

The pdf and the cdf of the log-Laplace distribution can be easily calculated as

$$
\begin{gathered}
f_{i}(x)= \begin{cases}\frac{\alpha_{i} \beta_{i}}{2} x^{\alpha_{i}-1}, & \text { if } x \leq \beta_{i}^{-1 / \alpha_{i}}, \\
\frac{\alpha_{i}}{2 \beta_{i}} x^{-\left(\alpha_{i}+1\right)}, & \text { if } x>\beta_{i}^{-1 / \alpha_{i}},\end{cases} \\
F_{i}(x)= \begin{cases}\frac{\beta_{i}}{2} x^{\alpha_{i}}, & \text { if } x \leq \beta_{i}^{-1 / \alpha_{i}}, \\
1-\frac{1}{2 \beta_{i}} x^{-\alpha_{i}}, & \text { if } x>\beta_{i}^{-1 / \alpha_{i}},\end{cases}
\end{gathered}
$$

respectively, where $x>0$. The reliability $R$ can be calculated from (1.1) by simple integration to yield

$$
R= \begin{cases}\frac{\alpha_{2}^{2} \beta_{2}^{\alpha_{1} / \alpha_{2}}}{2 \beta_{1}\left(\alpha_{2}^{2}-\alpha_{1}^{2}\right)}-\frac{\alpha_{1}^{2} \beta_{1}^{-\alpha_{2} / \alpha_{1}} \beta_{2}}{2\left(\alpha_{2}^{2}-\alpha_{1}^{2}\right)}, & \text { if } \beta_{1}^{-1 / \alpha_{1}} \leq \beta_{2}^{-1 / \alpha_{2}}, \\ 1-\frac{\alpha_{1}^{2} \beta_{1}^{\alpha_{2} / \alpha_{1}}}{2 \beta_{2}\left(\alpha_{1}^{2}-\alpha_{2}^{2}\right)}+\frac{\alpha_{2}^{2} \beta_{1} \beta_{2}^{-\alpha_{1} / \alpha_{2}}}{2\left(\alpha_{1}^{2}-\alpha_{2}^{2}\right)}, & \text { if } \beta_{1}^{-1 / \alpha_{1}}>\beta_{2}^{-1 / \alpha_{2}}\end{cases}
$$

The reliability $R$ for the random variables $X^{*}$ and $X^{* *}$ (having the $L_{B}$ system distribution and the $L_{U}$ system distribution, resp.) is the same as that for the log-Laplace 
distribution because

$$
\begin{aligned}
& \operatorname{Pr}\left(X_{1}^{*}<X_{2}^{*}\right)=\operatorname{Pr}\left(\frac{\exp \left(\left(Y_{1}-\log \left(\beta_{1}\right)\right) / \alpha_{1}\right)}{1+\exp \left(\left(Y_{1}-\log \left(\beta_{1}\right)\right) / \alpha_{1}\right)}<\frac{\exp \left(\left(Y_{2}-\log \left(\beta_{2}\right)\right) / \alpha_{2}\right)}{1+\exp \left(\left(Y_{2}-\log \left(\beta_{2}\right)\right) / \alpha_{2}\right)}\right) \\
& =\operatorname{Pr}\left(\exp \left(\frac{Y_{1}-\log \left(\beta_{1}\right)}{\alpha_{1}}\right)<\exp \left(\frac{Y_{2}-\log \left(\beta_{2}\right)}{\alpha_{2}}\right)\right) \\
& =\operatorname{Pr}\left(X_{1}<X_{2}\right) \text {, } \\
& \operatorname{Pr}\left(X_{1}^{* *}<X_{2}^{* *}\right)=\operatorname{Pr}\left(\sinh \left(\frac{Y_{1}-\log \left(\beta_{1}\right)}{\alpha_{1}}\right)<\sinh \left(\frac{Y_{2}-\log \left(\beta_{2}\right)}{\alpha_{2}}\right)\right) \\
& =\operatorname{Pr}\left(\exp \left(\frac{Y_{1}-\log \left(\beta_{1}\right)}{\alpha_{1}}\right)-\exp \left(-\frac{Y_{1}-\log \left(\beta_{1}\right)}{\alpha_{1}}\right)\right. \\
& \left.<\exp \left(\frac{Y_{2}-\log \left(\beta_{2}\right)}{\alpha_{2}}\right)-\exp \left(-\frac{Y_{2}-\log \left(\beta_{2}\right)}{\alpha_{2}}\right)\right) \\
& =\operatorname{Pr}\left(\exp \left(\frac{Y_{1}-\log \left(\beta_{1}\right)}{\alpha_{1}}\right)<\exp \left(\frac{Y_{2}-\log \left(\beta_{2}\right)}{\alpha_{2}}\right)\right) \\
& =\operatorname{Pr}\left(X_{1}<X_{2}\right) \text {. }
\end{aligned}
$$

We have used the fact that both $z /(1+z)$ and $z-1 / z$ are increasing functions of $z$.

\section{Acknowledgment}

The author is grateful to Professor Samuel Kotz (George Washington University, USA) and to Professor Marianna Pensky (University of Central Florida, USA) for introducing him to this area of research.

\section{References}

[1] G. Agrò, Maximum likelihood estimation for the exponential power function parameters, Comm. Statist. Simulation Comput. 24 (1995), no. 2, 523-536.

[2] D. F. Andrews, P. J. Bickel, F. R. Hampel, P. J. Huber, W. H. Rogers, and J. W. Tukey, Robust Estimates of Location: Survey and Advances, Princeton University Press, New Jersey, 1972.

[3] G. Aryal and S. Nadarajah, On the skew Laplace distribution, in preparation.

[4] N. Balakrishnan and S. Kocherlakota, On the double Weibull distribution: order statistics and estimation, Sankhyā Ser. B 47 (1985), no. 2, 161-178.

[5] O. Borghi, On a distribution of frequencies, Trabajos Estadíst. 16 (1965), 171-192 (Spanish).

[6] G. E. P. Box and G. C. Tiao, A further look at robustness via Bayes's theorem, Biometrika 49 (1962), 419-432.

[7] A. V. Dattatreya Rao and V. L. Narasimham, Linear estimation in double Weibull distribution, Sankhyā Ser. B 51 (1989), no. 1, 24-64.

[8] C. Fernández and M. F. J. Steel, On Bayesian modeling of fat tails and skewness, J. Amer. Statist. Assoc. 93 (1998), no. 441, 359-371.

[9] I. S. Gradshteyn and I. M. Ryzhik, Table of Integrals, Series, and Products, Academic Press, San Diego, 2000. 
[10] D. V. Hinkley and N. S. Revankar, A further analysis: "on the estimation of the Pareto law from underreported data", J. Econometrics 5 (1977), no. 1, 1-11.

[11] D. C. Hoaglin, F. Mosteller, and J. W. Tukey (eds.), Understanding Robust and Exploratory Data Analysis, John Wiley \& Sons, New York, 1983.

[12] M. S. Holla and S. K. Bhattacharya, On a compound Gaussian distribution, Ann. Inst. Statist. Math. 20 (1968), 331-336.

[13] P. N. Jones and G. J. McLachlan, Laplace-normal mixtures fitted to wind shear data, J. Appl. Stat. 17 (1990), 271-276.

[14] G. K. Kanji, A mixture model for wind shear data, J. Appl. Stat. 12 (1985), 49-58.

[15] R. R. L. Kantam and V. L. Narasimham, Linear estimation in reflected gamma distribution, Sankhyā Ser. B 53 (1991), no. 1, 25-47.

[16] S. Kapoor and G. K. Kanji, Application of the characterization theory to the mixture model, J. Appl. Stat. 17 (1990), 263-270.

[17] W. J. McGill, Random fluctuations of response rate, Psychometrika 27 (1962), 3-17.

[18] S. Nadarajah, Reliability for Logistic distributions, to appear in the International Journal of Engineering Simulation.

[19]_, Reliability for beta models, Serdica Math. J. 28 (2002), no. 3, 267-282.

[20]_, Reliability for extreme value distributions, Math. Comput. Modelling 37 (2003), no. 910, 915-922.

[21] Reliability for lifetime distributions, Math. Comput. Modelling 37 (2003), no. 7-8, 683688.

[22] S. Nadarajah and S. Kotz, Reliability for Pareto models, Metron 61 (2003), no. 2, 191-204.

[23] S. Poiraud-Casanova and C. Thomas-Agnan, About monotone regression quantiles, Statist. Probab. Lett. 48 (2000), no. 1, 101-104.

[24] A. P. Prudnikov, Yu. A. Brychkov, and O. I. Marichev, Integrals and Series. Vol. 1, Gordon and Breach Science Publishers, New York, 1986.

[25]_, Integrals and Series. Vol. 2, Gordon and Breach Science, New York, 1988.

[26] Integrals and Series. Vol. 3, Gordon and Breach Science, New York, 1990.

[27] A. J. Scallan, Maximum likelihood estimation for a normal/Laplace mixture distribution, The Statistician 41 (1992), 227-231.

[28] M. T. Subbotin, On the law of frequency of errors, Mat. Sb. 31 (1923), 296-300.

[29] P. R. Tadikamalla and N. L. Johnson, Systems of frequency curves generated by transformations of logistic variables, Biometrika 69 (1982), no. 2, 461-465.

[30] G. C. Tiao and D. R. Lund, The use of OLUMV estimators in inference robustness studies of the location parameters of a class of symmetric distributions, J. Amer. Statist. Assoc. 65 (1970), 371-386.

[31] A. Vasudeva Rao, A. V. Dattatreya Rao, and V. L. Narasimham, Optimum linear unbiased estimation of the scale parameter by absolute values of order statistics in the double exponential and the double Weibull distributions, Comm. Statist. Simulation Comput. 20 (1991), no. 4, $1139-1158$.

[32] S. Weerahandi and R. A. Johnson, Testing reliability in a stress-strength model when $X$ and $Y$ are normally distributed, Technometrics 34 (1992), no. 1, 83-91.

[33] R. Zeckhauser and M. Thompson, Linear regression with non-normal error terms, The Review of Economics \& Statistics 52 (1970), 280-286.

Saralees Nadarajah: Department of Mathematics, University of South Florida, Tampa, FL 336205700, USA

E-mail address: snadaraj@chuma1.cas.usf.edu 


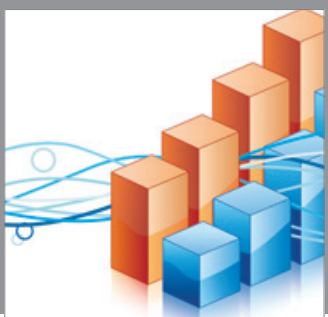

Advances in

Operations Research

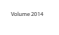

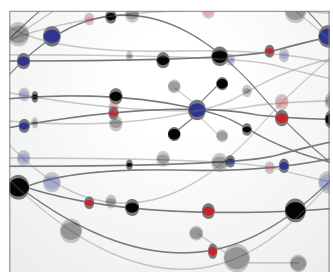

\section{The Scientific} World Journal
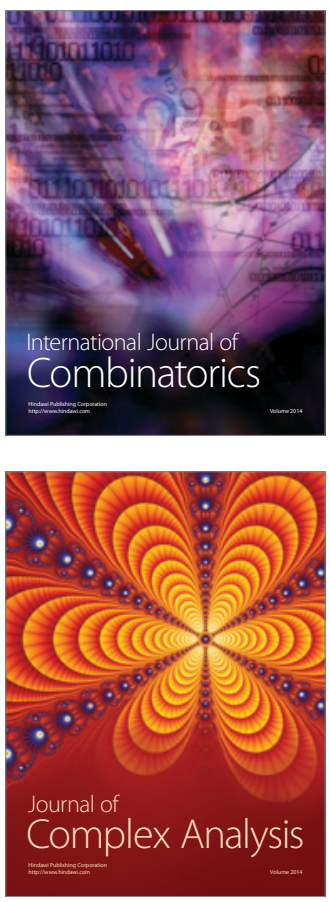

International Journal of

Mathematics and

Mathematical

Sciences
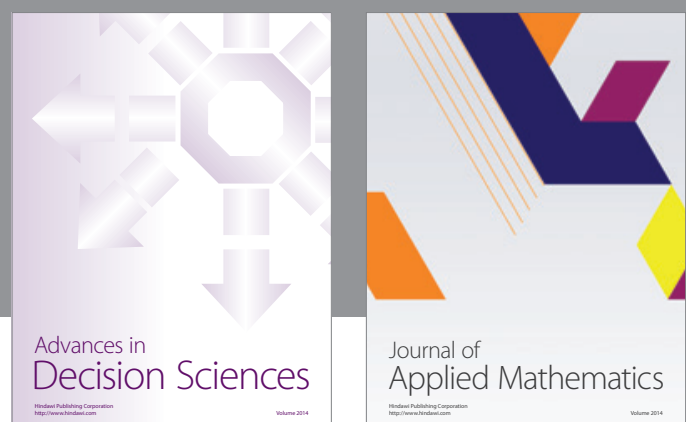

Journal of

Applied Mathematics
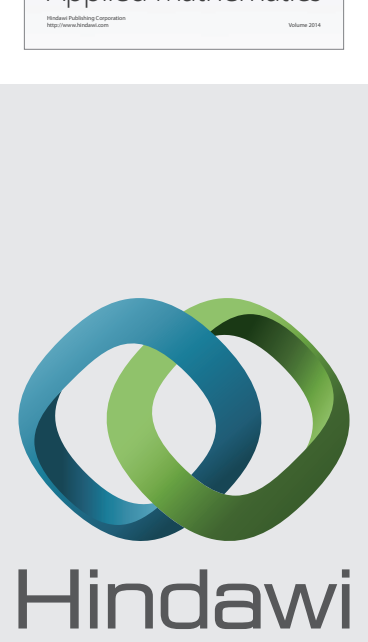

Submit your manuscripts at http://www.hindawi.com
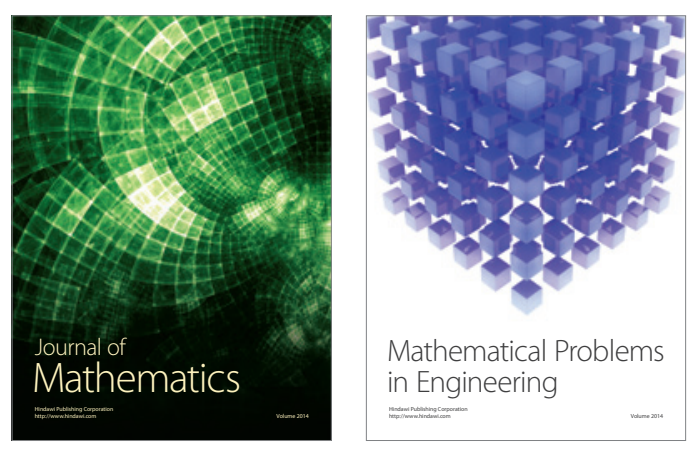

Mathematical Problems in Engineering
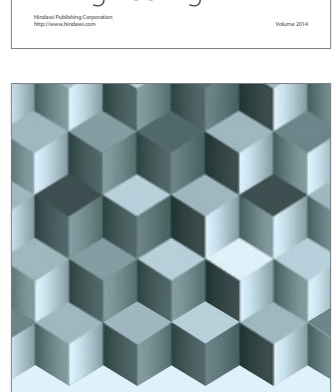

Journal of

Function Spaces
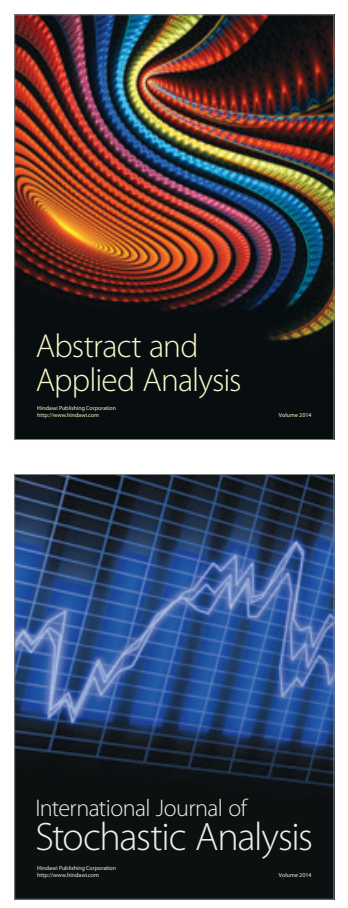

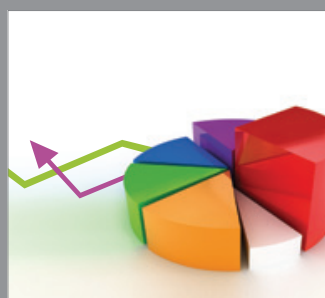

ournal of

Probability and Statistics

Promensencen
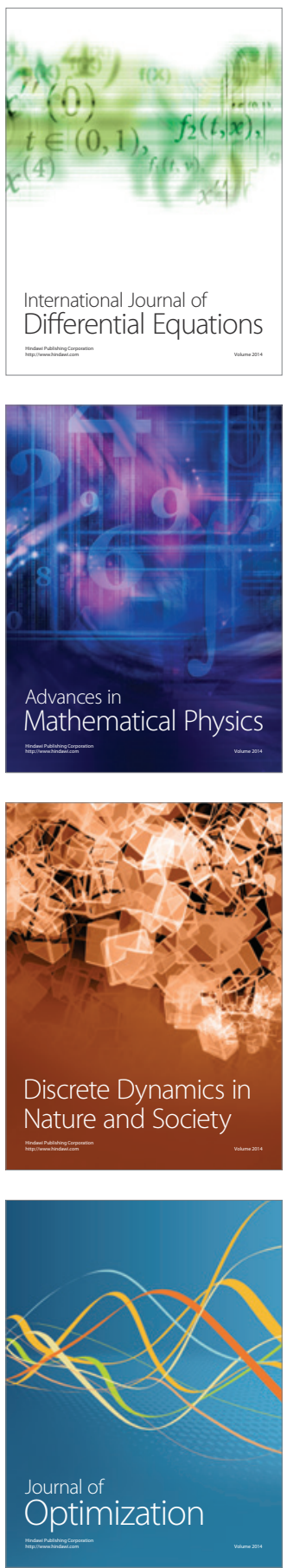